\title{
El análisis comparativo de la aplicación del sistema dual de aprendizaje en Universidades en Canadá con respecto al Instituto Tecnológico Superior de Puerto Vallarta para el desarrollo de habilidades de competitividad
}

\author{
Alejandra Medina Lozano, Instituto Tecnológico superior de Puerto Vallarta, México \\ Leticia Velarde Peña, Instituto Tecnológico superior de Puerto Vallarta, México
}

\begin{abstract}
Resumen: El mundo actual requiere a profesionistas competitivos, para lo cual las Universidades diseñan programas académicos pertinentes con prácticas en empresas del área geográfica donde se desarrollan. Con la finalidad de que los alumnos adquieran las competencias requeridas en el ámbito laboral. En el presente trabajo se realiza un análisis comparativo de la forma de aplicación del sistema dual de aprendizaje, sistema que tiene sus orígenes en Alemania en el cual los alumnos realizan prácticas en empresas de la región de su perfil profesional diseñadas, previstas y reguladas por la institución académica; dicho análisis se realiza con las características de aplicación del sistema en cuatro universidades de Canadá con respecto al Instituto Tecnológico Superior de Puerto Vallarta aplicado en prueba piloto, destacando las fortalezas y debilidades para determinar las áreas de oportunidad en el Instituto para orientar propuestas de aplicación tomando en consideración a Canadá como modelo debido que ocupa los primeros lugares de competitividad a nivel mundial .
\end{abstract}

Palabras clave: sistema dual de aprendizaje, prueba piloto, análisis comparativo, habilidades de competitividad

\begin{abstract}
In order for students to acquire the skills required in the workplace. The present work is a further investigation in which a comparative analysis of the application form dual apprenticeship system, a system that has its origins in Germany in which students complete internships at companies in the region designed professional profile, planned and regulated by the academic institution. This analysis is performed with the application characteristics of the system in five Canadian universities vs. Higher Technological Institute of Puerto Vallarta, highlighting the strengths and weaknesses to determine areas of opportunity in the Institute to guide implementation proposals considering Canada as a model because it occupies the top of global competitiveness.
\end{abstract}

Keywords: Dual Learning System, Pilot Trial, Comparative Analysis, Competitive Skills

\section{Introducción}

$\mathrm{H}$ oy día en México los jóvenes son los más afectados por el desempleo, revelado en el informe de mayo de 2014 del Instituto Nacional de Estadística y Geografía. (inegi, 2014) En éste se determina que si bien la tasa de desempleo en México es de $4.9 \%$, en el caso de los más jóvenes la cifra asciende a $8.44 \%$, de estos mismos el $80 \%$ tienen formación superior. Por lo tanto las universidades deben de generar programas y estrategias que propicien en ellos una formación integral mediante la cual se formen para su rápida inserción en la vida laboral en el área de profesionalización. El programa de formación dual, es una alternativa para Vincular las consideraciones teóricas en las escuelas de formación profesional con experiencias prácticas en empresa, basándonos en el mencionado programa que está diseñado bajo esta fundamentación denominado sistema dual de aprendizaje que surge en Alemania en 1973. "Mediante el programa se aporta a la economía no solo especialistas formados en el mundo práctico, sino que constituye una receta de éxito para transiciones satisfactorias al mundo laboral" (Euler,paq.4). En el mismo sentido (O'Higgins, 2001) menciona que es una ventaja del modelo dual el que se transfiera momentos del proceso de enseñanza-

Revista Internacional de Tecnologías en la Educación

Volumen 1, Número 2, 2014, <http://sobrelaeducacion.com>, ISSN 2386-8384

(C) Global Knowledge Academics. Alejandra Medina Lozano, Leticia Velarde

Peña. Todos los derechos reservados. Permisos: soporte@gkacademics.com 
aprendizaje a las empresas, porque esto es un factor que facilita la rápida inserción en el sector productivo a los estudiantes, por que modifica el estatus de egresado sin experiencia.

El sistema dual de aprendizaje: método de formación profesional para jóvenes de ambos sexos que aprenden la teoría de la ocupación en el centro de formación y la práctica en la empresa el cual brinda la oportunidad a los alumnos que tengan una buena base de estudios, de demostrar continuamente los conocimientos teóricos y habilidades adquiridas mediante su aplicación en tareas profesionales reales, y bajo diversos y cambiantes condicionantes del mundo laboral, dando la posibilidad de alternar la teoría con la práctica. Esto permite según (Aset, 1999) que los conocimientos se interrelacionen brindando un aprendizaje vivencial, combinando la formación práctica de la empresa con la formación teórica en una escuela profesional.

El principal objetivo en el plan de desarrollo nacional 2013-2018 es llevar a México a su máximo potencial por lo tanto en el apartado III. México con educación de calidad se proponen estrategias mediante las cuales las instituciones educativas creen los mecanismos necesarios para que los estudiantes de nivel profesional logren formación para la rápida inserción en el ámbito laboral y así se cumpla el principal objetivo del mencionado plan.

Se promoverá el trabajo conjunto de las autoridades escolares, maestros, alumnos y padres de familia, no sólo en el cumplimiento de planes y programas de estudio, sino en la conformación de verdaderas comunidades con metas compartidas y con el interés de brindar a los niños y jóvenes una educación que tampoco se limite al cumplimiento de lo esencial, sino que aspire a una formación integral. (Plan nacional de desarrollo 2013-2018, 2013)

Por su parte, Canadá no hay un plan de estudios nacional ni hay estándares nacionales para la educación, aunque nueve provincias y dos territorios están desarrollando un plan de estudios nacional. Datos obtenidos del Consejo de Ministros de Educación en Canadá, además todas las provincias están colaborando en fijar los estándares para la ciencia en lo referente al programa de los indicadores del logro de la escuela (SAIP) La educación superior se ofrece a través de instituciones públicas y privadas, las universidades se centran principalmente en los programas de grado, colegios comunitarios y técnicos, institutos de formación profesional que se centran en los programas de certificado y diploma, con la capacidad de otorgar títulos de pregrado. La educación superior también se proporciona a través del sistema de aprendizaje, La planificación del programa incorpora a negocios, industria y representantes de los trabajadores en los comités consultivos de la universidad.

La formación profesional se refiere a un programa de varios años, o una serie de cursos que ofrecen instrucción especializada en una técnica o un oficio con la intención de llevar al estudiante directamente en una carrera o un programa basado en la competencia o el comercio. De forma similar en Canadá existen universidades en las que en los programas de estudio prevé la realización de prácticas profesionales en las empresas. (Consejo Interamericano para el Desarrollo Integral , 2004). El método de aprendizaje sistema dual es usado con diferentes modalidades que se ajustan de acuerdo a las necesidades de la universidad, alumnos y el sector productivo.

En el presente artículo son los resultados de una investigación, La metodología de la realización del presente trabajo se baso en la búsqueda y análisis de la información contenida en las páginas web de 50 universidades en Canadá encontrando las características de aplicación del sistema dual en cuatro universidades, material con el que se fundamenta el comparativo realizado con la forma de aplicación de dicho modelo en el Instituto Tecnológico Superior de Puerto Vallarta, con el objetivo de destacar las fortalezas y debilidades en el proceso de aplicación en el Instituto permitiendo determinar las áreas de oportunidad, para partir de ellas y diseñar estrategias y rumbos de acción para reorientar propuestas de aplicación tomando en consideración a Canadá como modelo debido que ocupa los primeros lugares de competitividad a nivel mundial.

\section{Desarrollo}

En la Academy of Applied Pharmaceutical Sciences (Canada, 2014) los planes de estudios están diseñados considerando el uso software que ayuda a inferir los resultados de un entorno de trabajo real lo cual les da confianza a los alumnos en los conocimientos adquiridos, posterior mente realizan 
prácticas en empresas de la región en las cuales tienen convenios que aseguran la colocación a corto plazo de los egresados.

En el Instituto Tecnológico (Gonzalez Maldonado, 2013) no se cuenta con la infraestructura para el uso de simuladores, se realizan prácticas en empresas de la región donde se tienen convenios donde no se asegura colocación de los egresados, pero de acuerdo a la experiencia a la fecha desde que inicio el sistema dual en la institución ya han egresado tres generaciones de alumnos de los cuales el 96\% de los mismos están colocados en empleos afines a el área de formación.

En Ashton College (College, 2014) los estudiantes tienen la opción para obtener los conocimientos de perfil profesional el elegir entre tomar las clases regulares en un aula o salón de clases o la realización de prácticas en empresas, planeadas en coordinación de la universidad y las empresas mismas que son remuneradas, posterior a las practicas los alumnos realizan un resumen de la experiencia de aprendizaje adquirido en la empresa mismos que son validados por las universidades.

La universidad mantiene convenios con la industria para la colocación de los egresados, el estudiante debe mantener un vínculo con el coordinador para conocer sus preferencias laborales identificando las áreas de fortaleza de los estudiantes y los vincula con las empresas en el roster de Ashton. Así mismo proporciona asistencia en la personalización de los currículos, cartas de presentación y orientación para las entrevistas de los estudiantes para garantizar la colocación laboral.

En el Instituto Tecnológico (Gonzalez Maldonado, 2013) los alumnos en un inicio solo tenían la opción de adquirir sus conocimientos del perfil profesional en el aula y así mismo acreditarlos por medio de exámenes o realización de proyectos, a partir de la implementación del sistema dual de aprendizaje los alumnos adquieren conocimientos de su perfil profesional en las empresas y las acreditan por medio de exámenes de competencia laboral, realizan prácticas en la empresa que no son remuneradas por la misma pero reciben participación de las propinas que otorgan los clientes a los colaboradores del hotel. Para acreditar las asignaturas de la especialidad presentan al Instituto por medio de la educación a distancia resúmenes de experiencia de aprendizaje por medio de la plataforma moddle que servirán para validar los conocimientos para la adquisición de una especialización.

Con respecto al apoyo para la colocación laboral el instituto cuenta con una materia dentro de la retícula de los programas de estudios cuyo objetivo es orientar al alumno para conocer su perfil profesional, realización de curriculum y orientación para la entrevista de trabajo, así mismo se cuenta con bolsa de trabajo para alumnos y egresados del instituto tecnológico.

En Canadian Eastern Mennonite University (Eastern Mennonite University, 2014) ofrece dos tipos de cursos de práctica. Los cursos del primer tipo están programados junto con otros cursos durante el año académico. Estos cursos requieren que los estudiantes adquieran experiencia en las colocaciones individuales (un mínimo de sesenta horas por tres horas de crédito) y se reúnen regularmente en una clase práctica en la CMU. El segundo tipo, los cursos intensivos de práctica, requiere que los estudiantes pasan de un bloque de tiempo concentrado en una colocación en un mínimo de doce semanas (para un crédito de seis horas de duración). Los cursos de práctica más intensiva se desarrollan con programas específicos de CMU, pero no se limitan a los estudiantes que toman estos programas. La ayuda financiera está disponible para la práctica intensiva. Las ubicaciones apropiadas de práctica para cada alumno se arreglan con la oficina de Prácticas de acuerdo con los intereses individuales de los estudiantes y las metas académicas

En el Instituto (Gonzalez Maldonado, 2013) existe un programa de prácticas en el cual los alumnos asisten de 7:00 a 11:00 am a la escuela para recibir clases teóricas y de 12:00 a 18:00 hrs a la empresa a realizar prácticas en la empresa, dicho programa tiene una duración de 18 meses continuos tanto en la empresa como en la escuela, para la ubicación de las practicas están en contacto con el personal de capacitación de la empresa para que sean las mas apropiadas para cada uno de los alumnos de acuerdo a sus metas académicas, como ya se había mencionado los alumnos no tienen apoyo financiero por parte de la empresa solo se les proporciona una comida del día, uniforme y tienen acceso a el transporte de empleados de la empresa.

Otra institución en Canadá donde se cuenta con prácticas laborales es la Imperial Hotel Management College (Imperial Hotel Management College, 2014) en la cual se realizan con las siguientes características, el programa tiene una duración de 36 semanas en los campus de Hotel, los estudiantes 
que eligen la opción de vivir en el hotel tendrán el privilegio de disfrutar de la comodidad, conveniencia y lujo de vivir en una habitación de hotel de primera clase. Las clases se llevan a cabo en el sitio en los hoteles en las salas de reuniones de negocios, y los estudiantes obtienen experiencia de vida real de trabajo no sólo en el hotel donde tienen clases, sino también en los 'campus' otros hoteles seleccionados. El entorno seguro y acogedor de los hoteles hace que sea muy fácil para los estudiantes de combinar el estudio con el descubrimiento de la gente, la cultura y la belleza de Vancouver.

Los alumnos del Instituto que realizan prácticas solo acuden a ellas en el horario ya mencionado y no tienen la opción de vivir en el hotel.

\section{Conclusiones}

Posterior a los anteriores despliegues de características y comparativos entre las diferentes universidades de Canadá y el Instituto tecnológico se puede observar que el objetivo de los programas es el mismo, el que los alumnos adquieran conocimientos de la practica real en las empresas bajo un programa de prácticas planeadas por las Instituciones educativas en total acuerdo con las empresas de a cuerdo a las necesidades de ambas partes.

Que los alumnos logren la colocación laboral en cuanto egresan de la universidad en áreas afines a su formación profesional.

Otra similitud encontrada es que se les valida los conocimientos adquiridos en las empresas y forma parte de las competencias mínimas para la titulación de los alumnos en las universidades.

Las diferencias encontradas entre la aplicación de los diferentes programas tanto en las instituciones en Canadá con el Instituto es que en Canadá la realización de prácticas en las empresas les asegura la pronta colocación en las empresas en las cuales realizan las practicas, en cambio a los alumnos de ITS no es una certeza, esto se considera es un factor importante en para el éxito del programa ya que esto motiva a los alumnos en el desempeño de las mismas, así mismo el contar con una oferta laboral segura disminuye la incertidumbre del alumno, relajándose para concentrar sus esfuerzos en la realización de las practicas.

Otra diferencia encontrada entre los programas de prácticas en universidades de Canadá con el del instituto es el apoyo financiero que se otorga a los alumnos que realizan prácticas en empresas Canadienses, a diferencia de la empresa donde se realizan las prácticas los alumnos del Instituto Tecnológico, no proporcionan apoyo financiero, como apoyo les proporcionan una comida en el comedor de empleados, participación de las propinas que otorgan los huéspedes esta diferencia marca un factor de ventaja para las alumnos Canadienses en relación a los del Instituto, porque los alumnos con que cuenta el instituto en su mayoría son de clase media baja, donde los recursos económicos no son suficientes y por lo tanto posterior a las sesiones de clases en la escuela y las practicas en la empresa tienen que trabajar para hacerse de recursos financieros, lo que provoca un agotamiento en ellos, disminuyendo la productividad de los mismos.

Es observable que en las instituciones canadienses los alumnos tienen más beneficios por parte de las empresas provocando menor incertidumbre en los alumnos, factor importante que provoca productividad, competitividad y aprovechamiento en la realización de las prácticas reales en las empresas.

El Instituto Tecnológico superior de Puerto Vallarta posterior a este análisis comparativo debe de tomar acciones de vinculación con empresas para que otorgue certidumbre a los alumnos para la pronta colocación laboral en las empresas donde realizan las prácticas y la gestión de recursos financieros para los alumnos para que no tengan que trabajar en su tiempo de descanso y así aumenta la productividad y la competitividad en las prácticas y vida profesional. 


\section{REFERENCIAS}

Aset. (1999). Vocación por formar profesionales para el siglo XXI. Formacion Dual. Recuperado el 10 de julio de 1999, de htpp://www.tecladata.es/aset

Canada, I. (02 de febrero de 2014). Industri Canada. Recuperado el 01 de Abril de 2014, de http://www.ic.gc.ca/app/ccc/srch/nvgt.do?lang=eng\&prtl=1\&estblmntNo=234567100041\& profile $=$ cmpltPrfl\&profileId $=501 \&$ app $=$ sold

College, A. (2014). Ashton College. Recuperado el 12 de Marzo de 2014, de http://www.ashtoncollege.com/resource/program-advising/

Consejo Interamericano para el Desarrollo Integral . (2004). Educacion en Canada. Washinton, D.C: Comision Interamericana de Educación .

Eastern Mennonite University. (2014). Eastern Mennonite University. Recuperado el 12 de marzo de 2014, de http://www.emu.edu/

Euler, D. (2013). El Sistema Dual en Alemania- ¿Es posible transferir el modelo al estranjero? Alemania: Bertelsmann Stiftung.

Gonzalez Maldonado, C. E. (3 de Mayo de 2013). Licenciada en Mercadotecnia. (A. Medina Lozano, Entrevistador)

Imperial Hotel Management College. (2014). Imperial Hotel Management College. Recuperado el 12 de marzo de 2014, de http://imperialhotelmanagementcollege.com/

inegi. (abril de 2013). Instituto Nacional de Estadistica y Geografia. Recuperado el 03 de Julio de 2014, de http://www.inegi.org.mx/

O'Higgins, N. (2001). Desempeño juvenil y politica de empleo, una perpectiva global. Organizacion Internacional del Trabajo.

Plan nacional de desarrollo 2013-2018. (2013). Plan Nacional de Desarrollo, Gobierno de la Repiblica. Recuperado el 13 de Mayo de 2014, de http://pnd.gob.mx/

\section{SOBRE LAS AUTORAS}

Alejandra Medina Lozano: Docente De la institución en el área de Contabilidad, costos y finanzas. Investigadora educativa, miembro de la Red de posgrados en educación, miembro de la REDMIE, Ex becaria CONACIT, Perfil PROMEP, participante de proyectos de investigación Locales, Estatales y Nacionales.

Leticia Velarde Peña: Docente Investigador del Instituto Tecnológico Superior de Puerto Vallarta. Maestra en Tecnologías para el Aprendizaje. Ex-becario CONACYT, Perfil Promep. Miembro de REDMIIE, miembro de la red de posgrados en educación. Participante en proyectos de investigación Institucionales, estatales y Nacionales. Docente en educación a distancia. 